\title{
Complex Convexity of Orlicz-Lorentz Spaces and its Applications
}

\author{
by \\ Changsun CHOI, Anna KAMIŃSKA and Han Ju LEE \\ Presented by Aleksander PEŁCZYŃSKI
}

\begin{abstract}
Summary. We give sufficient and necessary conditions for complex extreme points of the unit ball of Orlicz-Lorentz spaces, as well as we find criteria for the complex rotundity and uniform complex rotundity of these spaces. As an application we show that the set of norm-attaining operators is dense in the space of bounded linear operators from $d_{*}(w, 1)$ into $d(w, 1)$, where $d_{*}(w, 1)$ is a predual of a complex Lorentz sequence space $d(w, 1)$, if and only if $w \in c_{0} \backslash \ell_{2}$.
\end{abstract}

1. Introduction. The notions of complex rotundity and uniform complex rotundity have been introduced in [22] and [10], respectively. The motivation came from vector-valued analytic function theory. In [22], it was proved that for any $X$-valued analytic function, the strong maximum modulus theorem holds true whenever $X$ is a complex rotund Banach space. Furthermore, in [22] it was shown that $L_{1}$ is complex rotund, and later on in [10] that it is also uniformly complex rotund. Characterizations of complex (uniform) rotundity of Orlicz or Musielak-Orlicz spaces were given in [6], while complex analogues of various moduli of convexity of a normed space were studied in [7] and [9].

In this paper, we find criteria for complex extreme points of the unit ball and for the complex rotundity and uniform complex rotundity of OrliczLorentz spaces. As corollaries we obtain the corresponding criteria for complex rotundity in Lorentz spaces. These results extend the already known criteria for real extreme points, rotundity and uniform rotundity in Lorentz or Orlicz-Lorentz spaces (cf. $[2,12,15,16,17])$. In the last section we apply

2000 Mathematics Subject Classification: 46E30, 46E20, 46G25.

Key words and phrases: Orlicz-Lorentz spaces, Marcinkiewicz spaces, complex extreme points, complex strict and uniform rotundity, norm attaining bilinear operators. 
complex rotundity of Lorentz sequence spaces to provide an answer to the problem raised in [1], in the case of complex spaces. In fact, employing the strong maximum modulus theorem in the complex Lorentz sequence space $d(w, 1)$, we show that the set of norm-attaining operators is dense in the space of bounded linear operators from $d_{*}(w, 1)$ into $d(w, 1)$, where $d_{*}(w, 1)$ is a predual of $d(w, 1)$, if and only if $w \in c_{0} \backslash \ell_{2}$.

Let $\mathbb{R}, \mathbb{R}_{+}, \mathbb{C}$ and $\mathbb{N}$ denote the sets of all real, nonnegative real, complex and natural numbers, respectively. We assume here that $\varphi: \mathbb{R}_{+} \rightarrow \mathbb{R}_{+}$is a Young function, that is, $\varphi$ is convex, $\varphi(0)=0$ and $\varphi(u)>0$ for $u>0$. A non-increasing function $w:[0, \gamma) \rightarrow(0, \infty)$ locally integrable with respect to the Lebesgue measure || on $[0, \gamma)$, where $\gamma \leq \infty$, is called a weight function. For $f:[0, \gamma) \rightarrow \mathbb{C}$ measurable with respect to || , define the distribution function $d_{f}(\theta)=|\{t \in[0, \gamma):|f(t)|>\theta\}|$ for $\theta \geq 0$, and its generalized inverse

$$
f^{*}(t)=\inf \left\{\theta>0: d_{f}(\theta) \leq t\right\}, \quad t \in[0, \gamma),
$$

called the decreasing rearrangement of $f$. The Orlicz-Lorentz space $\Lambda_{\varphi, w}$ is then defined as the set of all measurable functions $f:[0, \gamma) \rightarrow \mathbb{C}$ such that $\varrho_{\varphi}(\lambda f)<\infty$ for some $\lambda>0$, where

$$
\varrho_{\varphi}(f)=\int_{0}^{\gamma} \varphi\left(f^{*}(t)\right) w(t) d t=\int_{0}^{\gamma} \varphi\left(f^{*}\right) w .
$$

The space $\Lambda_{\varphi, w}$ equipped with the norm

$$
\|f\|=\inf \left\{\lambda>0: \varrho_{\varphi}(f / \lambda) \leq 1\right\}
$$

is a Banach space.

Now, let us define an Orlicz-Lorentz sequence space $\lambda_{\varphi, w}$. Given a complex-valued sequence $x=\{x(n)\}=\{x(n)\}_{n=1}^{\infty}$, consider the function $f(t)=$ $\sum_{k=1}^{\infty} x(k) \chi_{[k-1, k)}(t), t \geq 0$, and define a decreasing rearrangement of $x$ as follows:

$$
x^{*}(n)=f^{*}(n-1), \quad n \in \mathbb{N} .
$$

Let further $w=\{w(n)\}$ be a weight sequence, that is, $w(n)>0$ for all $n \in \mathbb{N}$, and $\{w(n)\}$ is non-increasing with $\sum_{n=1}^{\infty} w(n)=\infty$. Then analogously to the non-atomic case, the Orlicz-Lorentz sequence space $\lambda_{\varphi, w}$ consists of all sequences $x=\{x(n)\}$ such that for some $\lambda>0$,

$$
\varrho_{\varphi}(\lambda x)=\sum_{n=1}^{\infty} \varphi\left(\lambda x^{*}(n)\right) w(n)<\infty,
$$

and equipped with the norm $\|x\|=\inf \left\{\lambda>0: \varrho_{\varphi}(x / \lambda) \leq 1\right\}, \lambda_{\varphi, w}$ is a Banach space. Observe here that the condition $\sum_{n=1}^{\infty} w(n)=\infty$ implies that $\lambda_{\varphi, w} \hookrightarrow c_{0}$. 
If $\varphi(u)=u^{p}, 1 \leq p<\infty$, then $\Lambda_{\varphi, w}$ (resp., $\left.\lambda_{\varphi, w}\right)$ is a Lorentz space $\Lambda_{p, w}$ (resp., Lorentz sequence space $d(w, p))$ (cf. $[12,20]$ ) with the norm

$$
\|f\|=\left(\int_{0}^{\gamma}\left(f^{*}\right)^{p} w\right)^{1 / p} \quad\left(\operatorname{resp} .,\|x\|_{w, p}=\left(\sum_{n=1}^{\infty} x^{*}(n)^{p} w(n)\right)^{1 / p}\right) .
$$

The spaces $\Lambda_{\varphi, w}$ and $\lambda_{\varphi, w}$ coincide with the Orlicz space $L_{\varphi}$ and the Orlicz sequence space $\ell_{\varphi}$, respectively ([21]), whenever $w \equiv 1$.

The functional $\varrho_{\varphi}$ is a modular [21]. In particular $\varrho_{\varphi}$ is convex and orthogonally subadditive, that is, for any measurable functions $f, g$, we have $\varrho_{\varphi}(\alpha f+\beta g) \leq \alpha \varrho_{\varphi}(f)+\beta \varrho_{\varphi}(g)$ for $\alpha+\beta=1, \alpha, \beta \geq 0$ and $\varrho_{\varphi}(f+g) \leq$ $\varrho_{\varphi}(f)+\varrho_{\varphi}(g)$ if $\min \{|f|,|g|\}=0$.

We shall further need several growth conditions on $\varphi$ or $w$. We say that $\varphi$ satisfies the $\Delta_{2}^{w}$-condition if in the case of $\int_{0}^{\gamma} w<\infty$ (resp., $\left.\int_{0}^{\gamma} w=\infty\right)$ there exist $K \geq 1$ and $x_{0} \geq 0$ (resp., there is $K \geq 1$ ) such that

$$
\varphi(2 x) \leq K \varphi(x) \quad \text { for all } x \geq x_{0} \text { (resp. for all } x \geq 0 \text { ). }
$$

Recall also that $\varphi$ satisfies the $\delta_{2}$-condition if there exist $x_{0}>0$ and $K>0$ such that

$$
\varphi(2 x) \leq K \varphi(x) \quad \text { for all } 0 \leq x \leq x_{0}
$$

The weight function $w$ is regular if

$$
K=\inf _{0<x<\gamma / 2} \frac{\int_{0}^{2 x} w}{\int_{0}^{x} w}>1
$$

Most of the results collected in the next lemma are well known, and they correspond to the analogous results in Orlicz spaces [6]. We provide the proof here for the sake of completeness.

LEMMA 1.1. Suppose that $\varphi$ satisfies the $\Delta_{2}^{w}$-condition and let $f, f_{n}$ $\in \Lambda_{\varphi, w}$. Then:

(1) $\varrho_{\varphi}\left(f_{n}\right) \rightarrow \infty$ if and only if $\left\|f_{n}\right\| \rightarrow \infty$.

(2) For any $\varepsilon \in(0,1)$, there exists $\delta>0$ such that if $\varrho_{\varphi}(f) \leq 1-\varepsilon$ then $\|f\| \leq 1-\delta$.

(3) For any $\varepsilon \in(0,1)$, there exists $\delta>0$ such that if $\varrho_{\varphi}(f) \geq 1+\varepsilon$ then $\|f\| \geq 1+\delta$.

(4) $\varrho_{\varphi}\left(f_{n}\right) \rightarrow 1$ if and only if $\left\|f_{n}\right\| \rightarrow 1$.

(5) Norm and modular convergence are equivalent, i.e., $\varrho_{\varphi}\left(f_{n}\right) \rightarrow 0$ if and only if $\left\|f_{n}\right\| \rightarrow 0$.

Proof. (1) Suppose first that $\int_{0}^{\gamma} w=\infty$. Then the $\Delta_{2}^{w}$-condition is satisfied for all arguments. If $\left\{\left\|f_{n}\right\|\right\}$ is bounded, then there is $m \in \mathbb{N}$ such that $\left\|f_{n}\right\| \leq 2^{m}$ for all $n \in \mathbb{N}$. So $\varrho_{\varphi}\left(f_{n} / 2^{m}\right) \leq 1$ for all $n \in \mathbb{N}$, and thus for every $n \in \mathbb{N}$, 


$$
\varrho_{\varphi}\left(f_{n}\right) \leq K^{m} \varrho_{\varphi}\left(f_{n} / 2^{m}\right) \leq K^{m},
$$

which shows that $\left\{\varrho_{\varphi}\left(f_{n}\right)\right\}$ is bounded. Suppose now that $\int_{0}^{\gamma} w<\infty$. As above, if $\left\{\left\|f_{n}\right\|\right\}$ is bounded, then there is $m \in \mathbb{N}$ such that $\varrho_{\varphi}\left(f_{n} / 2^{m}\right) \leq 1$ for all $n \in \mathbb{N}$. Then for $x_{0}$ from the $\Delta_{2}^{w}$-condition and every $n \in \mathbb{N}$,

$$
\begin{aligned}
\varrho_{\varphi}\left(f_{n}\right) & \leq \varrho_{\varphi}\left(f_{n} \chi_{\left\{\left|f_{n}\right| \leq 2^{m} x_{0}\right\}}\right)+\varrho_{\varphi}\left(f_{n} \chi_{\left\{\left|f_{n}\right|>2^{m} x_{0}\right\}}\right) \\
& \leq \varphi\left(2^{m} x_{0}\right) \int_{0}^{\gamma} w+K^{m} \varrho_{\varphi}\left(f_{n} / 2^{m}\right) \\
& \leq \varphi\left(2^{m} x_{0}\right) \int_{0}^{\gamma} w+K^{m}<\infty .
\end{aligned}
$$

This proves that if $\varrho_{\varphi}\left(f_{n}\right) \rightarrow \infty$ then $\left\|f_{n}\right\| \rightarrow \infty$. The converse implication is obvious by definition of \|\| .

Suppose that (2) does not hold. Then there is $\varepsilon>0$ and a sequence $\left\{f_{n}\right\}$ such that $\varrho_{\varphi}\left(f_{n}\right)<1-\varepsilon$ and $1 / 2 \leq\left\|f_{n}\right\| \uparrow 1$. Let $L=\sup _{n}\left\{\varrho_{\varphi}\left(2 f_{n}\right)\right\}$ and $a_{n}=\left\|f_{n}\right\|^{-1}-1$. By (1), $L<\infty$. Then for every $n \in \mathbb{N}$,

$$
\begin{aligned}
1=\varrho_{\varphi}\left(f_{n} /\left\|f_{n}\right\|\right) & =\varrho_{\varphi}\left(2 a_{n} f_{n}+\left(1-a_{n}\right) f_{n}\right) \leq a_{n} \varrho_{\varphi}\left(2 f_{n}\right)+\left(1-a_{n}\right) \varrho_{\varphi}\left(f_{n}\right) \\
& \leq a_{n} L+1-\varepsilon \rightarrow 1-\varepsilon \quad \text { as } n \rightarrow \infty,
\end{aligned}
$$

which is a contradiction.

Suppose now that (3) does not hold. Then there are $\varepsilon>0$ and a sequence $\left\{f_{n}\right\}$ such that $\varrho_{\varphi}\left(f_{n}\right)>1+\varepsilon,\left\|f_{n}\right\| \downarrow 1$ and $1 \leq\left\|f_{n}\right\| \leq 2, n \in \mathbb{N}$. Setting now $b_{n}=\left\|f_{n}\right\| /\left(2\left\|f_{n}\right\|-1\right)$, we obtain

$$
\begin{aligned}
1+\varepsilon<\varrho_{\varphi}\left(f_{n}\right) & =\varrho_{\varphi}\left(b_{n} \frac{f_{n}}{\left\|f_{n}\right\|}+\left(1-b_{n}\right) 2 f_{n}\right) \\
& \leq b_{n} \varrho_{\varphi}\left(\frac{f_{n}}{\left\|f_{n}\right\|}\right)+\left(1-b_{n}\right) \varrho_{\varphi}\left(2 f_{n}\right) \\
& \leq b_{n}+\left(1-b_{n}\right) L \rightarrow 1 \quad \text { as } n \rightarrow \infty,
\end{aligned}
$$

which is a contradiction again. The last two claims are easy consequences of (1)-(3) and properties of $\varrho_{\varphi}$ and \|\| .

Remark 1.2. Notice that Lemma 1.1 also holds in Orlicz-Lorentz sequence spaces if we replace the $\Delta_{2}^{w}$-condition by the $\delta_{2}$-condition.

Finally, recall the following auxiliary results on geometric properties of Orlicz-Lorentz spaces.

Theorem $1.3([5,16])$. The following conditions are equivalent.

(1) $\varphi$ satisfies the $\Delta_{2}^{w}$-condition and $\int_{0}^{\gamma} w=\infty$ if $\gamma=\infty$ (resp., $\varphi$ satisfies the $\delta_{2}$-condition). 
(2) No isometric copy of $\ell_{\infty}$ is contained in $\Lambda_{\varphi, w}\left(\right.$ resp., $\left.\lambda_{\varphi, w}\right)$.

(3) $\Lambda_{\varphi, w}$ (resp., $\left.\lambda_{\varphi, w}\right)$ is separable.

Recall that a Banach space $X$ contains $\ell_{p}^{n}$ 's $\lambda$-uniformly or contains $\lambda$-uniformly copies of all $\ell_{p}^{n}$ 's (for $\lambda>1$ and $1 \leq p \leq \infty$ ) if for each $n \in \mathbb{N}$ there is an $n$-dimensional subspace $E$ of $X$ together with an isomorphism $U_{n}: \ell_{p}^{n} \rightarrow E$ such that $\left\|U_{n}\right\|\left\|U_{n}^{-1}\right\|<\lambda([8])$.

THEOREM 1.4 ([19]). Suppose that the weight function $w$ is not regular. Then $\Lambda_{\varphi, w}$ contains $\ell_{\infty}^{n}$ 's $\lambda$-uniformly for all $\lambda>1$.

A measurable function $f:[0, \infty) \rightarrow \mathbb{C}$ satisfies condition $(+)$ if there is $\theta_{1} \geq 0$ such that $|f(t)| \geq \theta_{1}$ a.e. and $d_{|f|-\theta_{1}}(\theta)<\infty$ for all $\theta>0$. Equivalently, $f$ satisfies condition $(+)$ if for all $\theta>0$ either $d_{f}(\theta)<\infty$ or $|\{t:|f(t)|<\theta\}|=0$.

LEMMA $1.5([15,16])$. Suppose that $f$ satisfies condition $(+)$ and there exists $A \subset[0, \gamma)$ with positive measure such that $|f(t)|<|g(t)|$ for all $t \in A$ and $|f| \leq|g|$. Then there exists a set $B$ with positive measure such that $f^{*}(t)<g^{*}(t)$ for $t \in B$.

2. Complex rotundity of Orlicz-Lorentz spaces. An element $x$ of the unit sphere $S_{X}$ of a complex Banach space $(X,\|\|)$ is a complex extreme point of the unit ball $B_{X}$ of $X$ if $\{x+\zeta y:|\zeta| \leq 1, \zeta \in \mathbb{C}\} \subset B_{X}$ for some $y \in X$ implies that $y=0$. A complex Banach space is complex rotund if every element of the unit sphere is a complex extreme point of its unit ball. It has been shown in [22] that $L_{\infty}$ or $\ell_{\infty}$ are not complex rotund, while $L_{1}$ or $\ell_{1}$ have this property. This clearly shows that complex rotundity and rotundity (in the usual real sense) are very different properties.

Now, we characterize the complex extreme points of the unit ball of $\Lambda_{\varphi, w}$.

TheOREM 2.1. A function $f \in \Lambda_{\varphi, w}$ is a complex extreme point of the unit ball of $\Lambda_{\varphi, w}$ if and only if the following conditions are satisfied:

(1) $\varrho_{\varphi}(f)=1$,

(2) $f$ satisfies condition $(+)$.

Proof. First recall [3] that for any measurable functions $h_{i}, i=1,2$, on $[0, \gamma)$ we have

$$
\int_{0}^{\gamma}\left(h_{1}+h_{2}\right)^{*} w \leq \int_{0}^{\gamma} h_{1}^{*} w+\int_{0}^{\gamma} h_{2}^{*} w .
$$

Now suppose that $f$ satisfies (1) and (2) and assume that $\|f+\zeta g\| \leq 1$ for 
all $|\zeta| \leq 1$ for some $g \in \Lambda_{\varphi, w}$. So, in view of the above inequality,

$$
\begin{aligned}
1=\int_{0}^{\gamma} \varphi\left(f^{*}\right) w & \leq \int_{0}^{\gamma} \varphi(|f|)^{*} w \leq \int_{0}^{\gamma}\left(\frac{\varphi(|f+\zeta g|)}{2}+\frac{\varphi(|f-\zeta g|)}{2}\right)^{*} w \\
& \leq \int_{0}^{\gamma}\left(\frac{\varphi(|f+\zeta g|)^{*}}{2}+\frac{\varphi(|f-\zeta g|)^{*}}{2}\right) w \leq 1 .
\end{aligned}
$$

Hence for any $|\zeta| \leq 1$,

$$
\varphi(|f|)^{*}=\left(\frac{\varphi(|f+\zeta g|)}{2}+\frac{\varphi(|f-\zeta g|)}{2}\right)^{*} .
$$

But by condition $(+)$ and Lemma 1.5, the last equality implies that for $|\zeta| \leq 1$

$$
\varphi(|f|)=\frac{1}{2}(\varphi(|f+\zeta g|)+\varphi(|f-\zeta g|)) \quad \text { a.e. }
$$

Without loss of generality we may assume that the equality above holds for all $t \in[0, \gamma)$. Using the convexity of $\varphi$, for each $t \in[0, \gamma)$, there exist $\alpha>0$ and $\beta \in \mathbb{R}$ such that

$$
\varphi(|f(t)|)=\alpha|f(t)|+\beta
$$

and for all $u \in \mathbb{R}_{+}$,

$$
\varphi(u) \geq \alpha u+\beta .
$$

For fixed $t \in[0, \gamma)$, there exist $\alpha>0$ and $\beta \in \mathbb{R}$ so that for all $|\zeta| \leq 1$,

$$
\begin{aligned}
\varphi(|f(t)|)=\alpha|f(t)|+\beta & =\frac{1}{2}(\varphi(|f(t)+\zeta g(t)|)+\varphi(|f(t)-\zeta g(t)|)) \\
& \geq \frac{1}{2}(\alpha|f(t)+\zeta g(t)|+\beta+\alpha|f(t)-\zeta g(t)|+\beta) .
\end{aligned}
$$

Hence for all $|\zeta| \leq 1$ and $t \in[0, \gamma)$,

$$
2|f(t)|=|f(t)+\zeta g(t)|+|f(t)-\zeta g(t)| .
$$

This implies that $g=0$ and proves the sufficiency.

Assume now that $f \in S_{\Lambda_{\varphi, w}}$ is a complex extreme point of $B_{\Lambda_{\varphi, w}}$. To show (1) suppose that $\varrho_{\varphi}(f)<1$ and $\|f\|=1$. By the definition of the norm, $\varrho_{\varphi}(r f)=\infty$ for all $r>1$. Setting $T=\operatorname{supp} f=\{t: f(t) \neq 0\}$ and $a=1-\varrho_{\varphi}(f)$, we have $a>0$. First we shall prove that there is $A_{0} \subset T$ so that $\left|A_{0}\right|>0$ and $\varrho_{\varphi}\left(f \chi_{A_{0}}\right)<a$, and $\varrho_{\varphi}\left(r f \chi_{A_{0}}\right)<\infty$ for all $r>1$. In fact, take $B_{n}$ so that $T=\bigcup_{n} B_{n}$ and $\left|B_{n}\right|<\infty$. We also have $T=\bigcup_{n} D_{n}$, where $D_{n}=\{t \in T:|f(t)| \leq n\}$. Clearly, $T=\bigcup_{n, m} B_{n} \cap D_{m}$. Since $|T|>0$, there exist $n, m \in \mathbb{N}$ such that $\left|D_{n} \cap B_{m}\right|>0$. Denoting by $D$ the set $D_{n} \cap B_{m}$ we have $0<|D|<\infty, D \subset T$, and

$$
\varrho_{\varphi}\left(f \chi_{D}\right) \leq \int_{0}^{\gamma} \varphi\left(n \chi_{D}\right)^{*} w=\int_{0}^{\gamma} \varphi(n) \chi_{[0,|D|)} w=\varphi(n) \int_{0}^{|D|} w<\infty,
$$


by the local integrability of $w$. By the absolute continuity of the integral, there exists $A_{0} \subset D$ such that

$$
\varrho_{\varphi}\left(f \chi_{A_{0}}\right) \leq \varphi(n) \int_{0}^{\left|A_{0}\right|} w<a
$$

For $r>1$ we also have

$$
\varrho_{\varphi}\left(r f \chi_{A_{0}}\right) \leq \varphi(r n) \int_{0}^{\left|A_{0}\right|} w<\infty .
$$

Continuity of the function $r \mapsto \varrho_{\varphi}\left(r f \chi_{A_{0}}\right), r>0$, yields $\varrho_{\varphi}\left(r_{1} f \chi_{A_{0}}\right)=a$ for some $r_{1}>1$. Choose $\delta>0$ so that $|z+1| \leq r_{1}$ for all $|z| \leq \delta$. Then setting $g=\delta f \chi_{A_{0}}$, we have for all $|\zeta| \leq 1$,

$$
\begin{aligned}
\varrho_{\varphi}(f+\zeta g) & =\varrho_{\varphi}\left(f \chi_{T \backslash A_{0}}+(1+\zeta \delta) f \chi_{A_{0}}\right) \\
& \leq \varrho_{\varphi}(f)+\varrho_{\varphi}\left(r_{1} f \chi_{A_{0}}\right)=\varrho_{\varphi}(f)+a=1 .
\end{aligned}
$$

Hence $\|f+\zeta g\| \leq 1$ for all $|\zeta| \leq 1$, where $g \neq 0$ a.e., so $f$ is not a complex extreme point.

In order to show (2) suppose that $f$ does not satisfy condition $(+)$. This clearly implies that $\gamma=\infty$. Then there is $\theta>0$ such that $d_{f}(\theta)=\infty$ and $|\{t:|f(t)|<\theta\}|>0$. Defining $A=\{t:|f(t)|<\theta\}$ we choose a set $A_{1} \subset A$ with positive measure and $a>0$ such that $|f(t)| \leq \theta-2 a$ for all $t \in A_{1}$. Letting $g(t)=a \chi_{A_{1}}(t)$, we have $(f+\zeta g)^{*}=f^{*}$ for all $|\zeta| \leq 1$. This shows that $f$ is not a complex extreme point and completes the proof.

Corollary 2.2. The complex Orlicz-Lorentz space $\Lambda_{\varphi, w}$ is complex rotund if and only if (i) $\varphi$ satisfies the $\Delta_{2}^{w}$-condition and (ii) $\int_{0}^{\infty} w=\infty$ if $\gamma=\infty$.

Proof. Suppose that (i) or (ii) does not hold. Then by Theorem 1.3, there is an isometric copy of $\ell_{\infty}$ contained in $\Lambda_{\varphi, w}$. Since $\ell_{\infty}$ is not complex rotund, neither is $\Lambda_{\varphi, w}$.

Suppose now that conditions (i) and (ii) are satisfied. Then by Lemma $1.1,\|f\|=1$ if and only if $\varrho_{\varphi}(f)=1$. If $\gamma<\infty$ then clearly every element in $\Lambda_{\varphi, w}$ satisfies condition $(+)$. Then by Theorem $2.1, \Lambda_{\varphi, w}$ is complex rotund. If $\gamma=\infty$, then for every $\theta>0$ and every $f \in \Lambda_{\varphi, w}$,

$$
\int_{0}^{\infty} \varphi\left(\theta\left(\chi_{\{t:|f(t)|>\theta\}}\right)^{*}\right) w=\varphi(\theta) \int_{0}^{|\{|f|>\theta\}|} w \leq \int_{0}^{\infty} \varphi(f)^{*} w<\infty .
$$

So $|\{t:|f(t)|>\theta\}|<\infty$. Therefore every element in $\Lambda_{\varphi, w}$ satisfies condition (+). Again Theorem 2.1 shows that $\Lambda_{\varphi, w}$ is complex rotund. This completes the proof. 
Corollary 2.3. An element of the unit sphere of a Lorentz space $\Lambda_{p, w}$, $1 \leq p<\infty$, is a complex extreme point if and only if it satisfies condition $(+)$.

Corollary 2.4. The Lorentz space $\Lambda_{p, w}, 1 \leq p<\infty$, is complex rotund if and only if $\int_{0}^{\infty} w=\infty$ when $\gamma=\infty$.

We finish this section with a result on complex rotundity in the sequence Orlicz-Lorentz space.

Theorem 2.5. The Orlicz-Lorentz sequence space $\lambda_{\varphi, w}$ is complex rotund if and only if $\varphi$ satisfies the $\delta_{2}$-condition.

Proof. If $\varphi$ does not satisfy the $\delta_{2}$-condition, then by Theorem 1.3 there is an isometric copy of $\ell_{\infty}$ in $\lambda_{\varphi, w}$, and so $\lambda_{\varphi, w}$ cannot be complex rotund. Conversely, suppose that $\varphi$ satisfies the $\delta_{2}$-condition. Take $x \in S_{\lambda_{\varphi, w}}$ and $y \in \lambda_{\varphi, w}$ such that $\|x+\zeta y\| \leq 1$ for all $|\zeta| \leq 1$. Then by the $\delta_{2}$-condition, Lemma 1.1 and Remark 1.2, $\varrho_{\varphi}(x)=1$. Moreover, there exists a permutation $\sigma: \mathbb{N} \rightarrow \mathbb{N}$ such that $x^{*}(n)=|x(\sigma(n))|$, since $\lim _{n \rightarrow \infty} x(n)=0$. Then, by the Hardy-Littlewood inequality [3] for each $|\zeta| \leq 1$,

$\sum_{n=1}^{\infty} \varphi(|x(\sigma(n))+\zeta y(\sigma(n))|) w(n) \leq \sum_{n=1}^{\infty} \varphi\left((x+\zeta y)^{*}(n)\right) w(n)=\varrho_{\varphi}(x+\zeta y) \leq 1$.

Hence for each $|\zeta| \leq 1$,

$$
\begin{aligned}
1 & =\varrho_{\varphi}(x)=\sum_{n=1}^{\infty} \varphi(|x(\sigma(n))|) w(n) \\
& \leq \frac{1}{2} \sum_{n=1}^{\infty}(\varphi(|x(\sigma(n))+\zeta y(\sigma(n))|)+\varphi(|x(\sigma(n))-\zeta y(\sigma(n))|)) w(n) \leq 1 .
\end{aligned}
$$

This implies that for each $|\zeta| \leq 1$ and $n \in \mathbb{N}$,

$$
\varphi(|x(\sigma(n))|)=\frac{1}{2}(\varphi(|x(\sigma(n))+\zeta y(\sigma(n))|)+\varphi(|x(\sigma(n))-\zeta y(\sigma(n))|)) .
$$

By the same argument as in the proof of Theorem 2.1, we infer that $y=0$ and this completes the proof.

Corollary 2.6. The Lorentz sequence space $d(w, p)$ is complex rotund for all $1 \leq p<\infty$.

3. Uniform complex rotundity of Orlicz-Lorentz spaces. We say that a complex Banach space $(X,\|\|)$ is uniformly complex rotund, called further uniformly c-convex, if for any $\varepsilon>0$, there exists $\delta=\delta(\varepsilon)>0$ such that if $x, y \in X,\|y\|>\varepsilon$ and $\|x+\zeta y\| \leq 1$ for all $|\zeta| \leq 1$ then $\|x\| \leq 1-\delta$. Notice that every uniformly convex complex Banach space is 
uniformly $c$-convex. But in general the converse is not true, since it is well known that $L_{1}$ is uniformly $c$-convex [10].

The following moduli of complex convexity for a Banach space $X$ were introduced in [7]. For $0<p<\infty$ and $0 \leq \varepsilon \leq 2$, we define

$$
\begin{aligned}
& H_{p}^{X}(\varepsilon)=\inf \left\{\left(\frac{1}{2 \pi} \int_{0}^{2 \pi}\left\|x+e^{i \theta} y\right\|^{p} d \theta\right)^{1 / p}-1:\|x\|=1,\|y\|=\varepsilon\right\}, \\
& H_{\infty}^{X}(\varepsilon)=\inf \left\{\sup \left\{\left\|x+e^{i \theta} y\right\|: 0 \leq \theta \leq 2 \pi\right\}-1:\|x\|=1,\|y\|=\varepsilon\right\} .
\end{aligned}
$$

Let $f$ and $g$ be non-negative, non-decreasing functions on $[0,2]$. We shall write $f \preccurlyeq g$ if there is $K \geq 1$ such that $f(\varepsilon / K) \leq K g(\varepsilon)$ for all $0<\varepsilon<1 / K$. We write $f \sim g$ ( $f$ and $g$ are then said to be equivalent at zero) if $f \preccurlyeq g$ and $g \preccurlyeq f$. It has been showed in [7] that for $0<p<\infty$, the moduli $H_{p}^{X}$ are all equivalent at zero.

We shall say that a complex Banach space $(X,\|\|)$ is uniformly $P L$ convex (resp., uniformly $H_{\infty}^{X}$-convex) if $H_{1}^{X}(\varepsilon)>0$ for all $\varepsilon>0$ (resp., $H_{\infty}^{X}(\varepsilon)>0$ for all $\left.\varepsilon>0\right)$. It is clear that $X$ is uniformly $c$-convex if and only if it is $H_{\infty}^{X}$-convex. Moreover, $H_{\infty}^{X}(\varepsilon) \geq H_{1}^{X}(\varepsilon)$, and in [9] it was showed that there exists $A>0$ such that for every complex Banach space $X$, $A\left(H_{\infty}^{X}(\varepsilon)\right)^{2} \leq H_{1}^{X}(\varepsilon)$ for all $0<\varepsilon \leq 1$. This implies that for complex Banach spaces the notions of uniform $c$-convexity, uniform $H_{\infty}^{X}$-convexity and uniform $P L$-convexity coincide.

We will next investigate uniform $c$-convexity of Orlicz-Lorentz spaces. Recall the following useful facts about uniform $c$-convexity.

LEMma 3.1 ([6]). Let $X$ be a complex Banach space. Then the following conditions are equivalent.

(1) $X$ is uniformly c-convex.

(2) For every $\varepsilon>0$, there is $\delta(\varepsilon)>0$ such that $x, y \in X,\|y\|>\varepsilon$, $\|x+\zeta y\| \leq 1$ for all $\zeta= \pm 1, \pm i$ implies $\|x\| \leq 1-\delta(\varepsilon)$.

(3) For every $\varepsilon>0$, there is $\delta(\varepsilon)>0$ such that for any $x, y \in X$,

$$
\|y\| \geq \varepsilon \max \{\|x+y\|,\|x-y\|,\|x+i y\|,\|x-i y\|\}
$$

implies

$$
\|x\| \leq \frac{1-\delta(\varepsilon)}{4}(\|x+y\|+\|x-y\|+\|x+i y\|+\|x-i y\|) .
$$

Notice that $\mathbb{C}$ with the absolute value as a norm is a uniformly $c$-convex space.

The following lemma will allow us to reduce the investigations of the norm in $\Lambda_{\varphi, w}$ to the modular $\varrho_{\varphi}$.

Lemma 3.2. Suppose $\varphi$ satisfies the $\Delta_{2}^{w}$-condition. Then the OrliczLorentz space $\Lambda_{\varphi, w}$ is uniformly c-convex if and only if $\varrho_{\varphi}$ is uniformly 
c-convex, i.e. for any $\varepsilon>0$, there exists $\delta=\delta(\varepsilon)>0$ such that if $f, g \in \Lambda_{\varphi, w}$ and

$$
\varrho_{\varphi}(g)>\varepsilon, \quad \varrho_{\varphi}(f+\zeta g) \leq 1 \quad \text { for all }|\zeta| \leq 1
$$

(or equivalently for all $\zeta= \pm 1, \pm i$ ), then $\varrho_{\varphi}(f) \leq 1-\delta$.

Proof. Suppose $\varphi$ satisfies the $\Delta_{2}^{w}$-condition and assume $\Lambda_{\varphi, w}$ is not uniformly $c$-convex. Then there are $\varepsilon_{0}>0$ and sequences $\left\{f_{n}\right\},\left\{g_{n}\right\}$ in $\Lambda_{\varphi, w}$ so that for every $n \in \mathbb{N}$ and every $|\zeta| \leq 1$,

$$
\left\|f_{n}+\zeta g_{n}\right\| \leq 1, \quad\left\|g_{n}\right\| \geq \varepsilon_{0}, \quad \lim _{n}\left\|f_{n}\right\|=1
$$

By definition of \|\| it follows that $\varrho_{\varphi}\left(f_{n}+\zeta g_{n}\right) \leq 1$. Moreover, by Lemma 1.1(4), $\lim _{n} \varrho_{\varphi}\left(f_{n}\right)=1$, and by Lemma 1.1(5), there exists $\varepsilon_{1}>0$ such that $\varrho_{\varphi}\left(g_{n}\right) \geq \varepsilon_{1}$ for every $n \in \mathbb{N}$. So $\varrho_{\varphi}$ is not uniformly $c$-convex. The converse can be proved by a similar argument.

For a regular weight $w$ we obtain the following lemma (cf. [12]).

LEMma 3.3. Suppose that the weight function $w$ is regular, so that

$$
K=\inf _{0<x<\gamma / 2} \frac{\int_{0}^{2 x} w}{\int_{0}^{x} w}>1 .
$$

Then for fixed $m \in \mathbb{N}$, there is $\theta=\theta(m)>0$ such that for every $\sigma \in$ $\left(0, \gamma /\left(2^{m}+1\right)\right)$,

$$
\int_{2^{m} \sigma}^{\left(2^{m}+1\right) \sigma} w \geq \theta \int_{0}^{\sigma} w .
$$

If $\gamma<\infty$ then for each $0<a<1$, there is $\theta=\theta(a)>0$ such that for every $\sigma \in[a \gamma, \gamma]$,

$$
\int_{\gamma-\sigma}^{\gamma} w \geq \theta \int_{0}^{\sigma} w
$$

Proof. Let $0<\sigma<\gamma / 2^{m+1}$. Since $w$ is non-increasing,

Then

$$
\int_{2^{m} \sigma}^{\left(2^{m}+1\right) \sigma} w \geq \frac{1}{2^{m}} \int_{2^{m} \sigma}^{2^{m+1} \sigma} w .
$$

$$
\begin{aligned}
\frac{\int_{2^{m} \sigma}^{\left(2^{m}+1\right) \sigma} w}{\int_{0}^{\sigma} w} & =\frac{\left.\int_{2^{m} \sigma}^{\left(2^{m}\right.}+1\right) \sigma}{\int_{0}^{2^{m} \sigma} w} \frac{\int_{0}^{2^{m} \sigma} w}{\int_{0}^{\sigma} w} \geq K^{m} \frac{\int_{2^{m} \sigma}^{\left(2^{m}+1\right) \sigma} w}{\int_{0}^{2^{m} \sigma} w} \\
& \geq\left(\frac{K}{2}\right)^{m} \frac{\int_{2^{m} \sigma}^{2^{m+1} \sigma} w}{\int_{0}^{2^{m} \sigma} w}=\left(\frac{K}{2}\right)^{m} \frac{\int_{0}^{2^{m+1} \sigma} w-\int_{0}^{2^{m} \sigma} w}{\int_{0}^{2^{m} \sigma} w} \\
& \geq\left(\frac{K}{2}\right)^{m}(K-1)>0 .
\end{aligned}
$$


So $\theta=(K / 2)^{m}(K-1)>0$ satisfies (3.2) if $\gamma=\infty$. Now if $\gamma<\infty$ and $\gamma / 2^{m+1} \leq \sigma<\gamma /\left(2^{m}+1\right)$, then $\theta=\min \left\{(K / 2)^{m}(K-1), \int_{\left(1-2^{-m-1}\right) \gamma}^{\gamma} w / \int_{0}^{\gamma} w\right\}$ $>0$ satisfies (3.2) if $\gamma<\infty$. For the last claim, if $\gamma<\infty$ then notice that the function $\sigma \mapsto \int_{\gamma-\sigma}^{\gamma} w / \int_{0}^{\sigma} w$ is positive and continuous on $[a \gamma, \gamma]$. So there is $\theta=\theta(a)>0$ such that for every $a \gamma \leq \sigma \leq \gamma$,

$$
\int_{\gamma-\sigma}^{\gamma} w \geq \theta \int_{0}^{\sigma} w
$$

which completes the proof.

TheOREM 3.4. The Orlicz-Lorentz space $\Lambda_{\varphi, w}$ is uniformly c-convex if and only if the following conditions are fulfilled:

(1) $\varphi$ satisfies the $\Delta_{2}^{w}$-condition;

(2) the weight function $w$ is regular.

Proof. Suppose that $\varphi$ satisfies the $\Delta_{2}^{w}$-condition and $w$ is regular, but $\Lambda_{\varphi, w}$ is not uniformly $c$-convex. By Lemma $3.2, \varrho_{\varphi}$ is not uniformly $c$-convex. So there are $\varepsilon>0$ and sequences $\left\{f_{n}\right\},\left\{g_{n}\right\}$ in $\Lambda_{\varphi, w}$ so that for every $n \in \mathbb{N}$ and every $|\zeta| \leq 1$,

$$
\varrho_{\varphi}\left(f_{n}+\zeta g_{n}\right) \leq 1, \quad \varrho_{\varphi}\left(g_{n}\right) \geq 2 \varepsilon, \quad \lim _{n} \varrho_{\varphi}\left(f_{n}\right)=1 .
$$

By Theorem 1.3, $\Lambda_{\varphi, w}$ is separable. Then the family of all simple functions with supports of finite measure is dense in $\Lambda_{\varphi, w}$. So we may assume that for each $n \in \mathbb{N}$ there is $\xi_{n}>0$ so that both $f_{n}$ and $g_{n}$ are constant on each interval of the form $\left[(k-1) \xi_{n}, k \xi_{n}\right) \in[0, \gamma), k \in \mathbb{N}$, and both $f_{n}$ and $g_{n}$ have supports of finite measure.

For $n \in \mathbb{N}$, let

$$
E_{n}=\left\{t \in[0, \gamma):\left|g_{n}(t)\right| \geq \frac{\varepsilon}{4} \max \left\{\left|f_{n}(t)+\zeta g_{n}(t)\right|: \zeta= \pm 1, \pm i\right\}\right\} .
$$

Then

$$
\varrho_{\varphi}\left(g_{n} \chi_{E_{n}^{\mathrm{c}}}\right) \leq \frac{\varepsilon}{4} \sum_{\zeta= \pm 1, \pm i} \varrho_{\varphi}\left(f_{n}+\zeta g_{n}\right) \leq \varepsilon
$$

where $E_{n}^{\mathrm{c}}=[0, \gamma) \backslash E_{n}$. Hence $2 \varepsilon \leq \varrho_{\varphi}\left(g_{n}\right) \leq \varrho_{\varphi}\left(g_{n} \chi_{E_{n}}\right)+\varrho_{\varphi}\left(g_{n} \chi_{E_{n}^{\mathrm{c}}}\right)$ implies that

$$
\varrho_{\varphi}\left(g_{n} \chi_{E_{n}}\right) \geq \varepsilon
$$

Setting

$$
s_{n}=\frac{1}{4} \sum_{\zeta= \pm 1, \pm i} \varphi\left(\left|f_{n}+\zeta g_{n}\right|\right)=\frac{1}{4} \sum_{\zeta= \pm 1, \pm i} \varphi\left(\left|g_{n}+\zeta f_{n}\right|\right),
$$

by the uniform convexity of $\mathbb{C}$ there is $\delta_{1}>0$ such that for all $n \in \mathbb{N}$ and all $t \in E_{n}$, 


$$
\varphi\left(\left|f_{n}(t)\right|\right) \leq \frac{1-\delta_{1}}{4} \sum_{\zeta= \pm 1, \pm i} \varphi\left(\left|f_{n}(t)+\zeta g_{n}(t)\right|\right)=\left(1-\delta_{1}\right) s_{n}(t) .
$$

Notice that for every $t \in[0, \gamma)$,

$$
\varphi\left(\left|f_{n}(t)\right|\right) \leq s_{n}(t), \quad \varphi\left(\left|g_{n}(t)\right|\right) \leq s_{n}(t) .
$$

Let

$$
h_{n}=\left(1-\delta_{1}\right) s_{n} \chi_{E}+\varphi\left(\left|f_{n}\right|\right) \chi_{E_{n}^{c}} .
$$

We use here the method invented in [12]. For convenience, we further omit the index $n$. Thus, until the end of the proof, $f, g, h, s, E$ and $\xi$ will stand for $f_{n}, g_{n}, h_{n}, s_{n}, E_{n}$ and $\xi_{n}$, respectively. Since $f$ and $g$ are constant on each interval $[(k-1) \xi, k \xi)$ and have supports of finite measure, it follows that $h$ as well as $h \chi_{E}$ are constant on each interval $[(k-1) \xi, k \xi)$ and have a finite number of values. Thus, for some $j, r \in \mathbb{N}$,

$$
\begin{aligned}
& h^{*}=\sum_{i=1}^{j} h^{i} \chi_{[(i-1) \xi, i \xi)}, \quad\left(h \chi_{E}\right)^{*}=\sum_{k=1}^{r} h^{i_{k}} \chi_{[(k-1) \xi, k \xi)}, \\
& \left(s \chi_{E}\right)^{*}=\frac{1}{1-\delta_{1}} \sum_{k=1}^{r} h^{i_{k}} \chi_{[(k-1) \xi, k \xi)}=\sum_{k=1}^{r} s^{k} \chi_{[(k-1) \xi, k \xi)},
\end{aligned}
$$

where $h^{1} \geq \ldots \geq h^{j}>0, h^{i_{1}} \geq \ldots \geq h^{i_{r}}>0$ and $s^{k}=\frac{1}{1-\delta_{1}} h^{i_{k}}$. Notice that for each $t \in\left[\left(i_{k}-1\right) \xi, i_{k} \xi\right)$,

$$
h^{*}(t)+\frac{\delta_{1}}{1-\delta_{1}} h^{*}(t)=h^{i_{k}}+\frac{\delta_{1}}{1-\delta_{1}} h^{i_{k}}=s^{k} .
$$

Hence by the Hardy-Littlewood inequality [3],

$$
\begin{aligned}
& \int_{0}^{\gamma} h^{*} w+\frac{\delta_{1}}{1-\delta_{1}} \sum_{k=1}^{r} \int_{\left(i_{k}-1\right) \xi}^{i_{k} \xi} h^{*} w \\
& =\sum_{k=1}^{r} \int_{\left(i_{k}-1\right) \xi}^{i_{k} \xi}\left(h^{*} w+\frac{\delta_{1}}{1-\delta_{1}} h^{*} w\right)+\sum_{\substack{j \in\{1, \ldots, n\} \\
j \notin\left\{i_{1}, \ldots, i_{r}\right\}}} \int_{\substack{j \xi \\
j}}^{j \xi} h^{*} w \\
& \leq \sum_{k=1}^{r} s^{k} \int_{\left(i_{k}-1\right) \xi}^{i_{k} \xi} w+\sum_{\substack{j \in\{1, \ldots, n\} \\
j \notin\left\{i_{1}, \ldots, i_{r}\right\}}} \int_{\substack{j \xi \\
(j-1) \xi}}^{j \xi} s^{*} w \\
& \leq \int_{0}^{\gamma} s^{*} w=\int_{0}^{\gamma} \frac{1}{4}\left(\sum_{\zeta= \pm 1, \pm i} \varphi(|f+\zeta g|)^{*} w\right) \\
& =\frac{1}{4} \sum_{\zeta= \pm 1, \pm i} \varrho_{\varphi}(|f+\zeta g|) \leq 1 \text {. }
\end{aligned}
$$


Since $h^{*} \geq \varphi(|f|)^{*}$,

$$
\int_{0}^{\gamma} h^{*} w \geq \varrho_{\varphi}(f) \rightarrow 1
$$

Thus

$$
\sum_{k=1}^{r} \int_{\left(i_{k}-1\right) \xi}^{i_{k} \xi} h^{*} w \rightarrow 0
$$

Moreover, in view of (3.4) we get

$$
\begin{aligned}
\int_{0}^{\gamma}\left(h \chi_{E}\right)^{*} w & =\left(1-\delta_{1}\right) \int_{0}^{\gamma}\left(s \chi_{E}\right)^{*} w \\
& \geq\left(1-\delta_{1}\right) \int_{0}^{\gamma} \varphi\left(|g| \chi_{E}\right)^{*} w=\left(1-\delta_{1}\right) \varrho_{\varphi}\left(g \chi_{E}\right) \geq\left(1-\delta_{1}\right) \varepsilon .
\end{aligned}
$$

By regularity of $w$, suppose further that $K>1$ is a constant in (3.1).

Applying the definition of $h$, in view of (3.6) and (3.7), and letting $\varepsilon_{3}=$ $\left(1-\delta_{1}\right) \varepsilon>0, \alpha^{k}=h^{i_{k}}, \beta^{r}=\alpha^{r}$, and $\beta^{k}=\alpha^{k}-\alpha^{k+1}$ for $k<r$, we obtain the following formulas:

$$
\begin{aligned}
\beta^{1} \int_{0}^{i_{1} \xi} w+\beta^{2} & \int_{0}^{i_{2} \xi} w+\ldots+\beta^{r} \int_{0}^{i_{r} \xi} w \\
& =\int_{0}^{i_{1} \xi} h^{i_{1}} w+\int_{i_{1} \xi}^{i_{2} \xi} h^{i_{2}} w+\ldots+\int_{i_{r-1} \xi}^{i_{r} \xi} h^{i_{r}} w \leq \int_{0}^{\gamma} h^{*} w \leq 1,
\end{aligned}
$$

$$
\beta^{1} \int_{0}^{\xi} w+\beta^{2} \int_{0}^{2 \xi} w+\ldots+\beta^{r} \int_{0}^{r \xi} w=\int_{0}^{\gamma}\left(h \chi_{E}\right)^{*} w \geq \varepsilon_{3}>0
$$

$$
\beta^{1} \int_{\left(i_{1}-1\right) \xi}^{i_{1} \xi} w+\ldots+\beta^{r}\left(\int_{\left(i_{1}-1\right) \xi}^{i_{1} \xi} w+\ldots+\int_{\left(i_{r}-1\right) \xi}^{i_{r} \xi} w\right)=\sum_{k=1}^{r} \int_{\left(i_{k}-1\right) \xi}^{i_{k} \xi} h^{*} w \rightarrow 0
$$

Choose $m$ so that $K^{m} \varepsilon_{3} / 2>1$. Now in view of (3.8) and regularity of $w$,

$$
1 \geq \sum_{i_{k}>2^{m} k} \beta^{k} \int_{0}^{i_{k} \xi} w \geq \sum_{i_{k}>2^{m} k} \beta^{k} \int_{0}^{2^{m} k \xi} w \geq K^{m} \sum_{i_{k}>2^{m} k} \beta^{k} \int_{0}^{k \xi} w
$$

where $k=1, \ldots, r$. This implies that

$$
\sum_{i_{k}>2^{m} k} \beta^{k} \int_{0}^{k \xi} w \leq \frac{1}{K^{m}}<\frac{\varepsilon_{3}}{2} .
$$


Hence by (3.9),

$$
\sum_{i_{k} \leq 2^{m} k} \beta^{k} \int_{0}^{k \xi} w \geq \frac{\varepsilon_{3}}{2} .
$$

Assume first that $\gamma=\infty$. In view of monotonicity of $w$, by Lemma 3.3, there is $\theta>0$ depending only on $m$, such that

$$
\begin{aligned}
\sum_{i_{k} \leq 2^{m} k} \beta^{k}\left(\int_{\left(i_{1}-1\right) \xi}^{i_{1} \xi} w+\ldots+\int_{\left(i_{k}-1\right) \xi}^{i_{k} \xi} w\right) \\
\geq \sum_{i_{k} \leq 2^{m} k} k \beta^{k} \int_{\left(i_{k}-1\right) \xi}^{i_{k} \xi} w \geq \sum_{i_{k} \leq 2^{m} k} k \beta^{k} \int_{2^{m} k \xi}^{\left(2^{m} k+1\right) \xi} w \\
\geq \sum_{i_{k} \leq 2^{m} k} \beta^{k} \int_{2^{m} k \xi}^{\left(2^{m}+1\right) k \xi} w \geq \sum_{i_{k} \leq 2^{m} k} \theta \beta^{k} \int_{0}^{k \xi} w \geq \frac{\theta \varepsilon_{3}}{2} .
\end{aligned}
$$

But this contradicts (3.10), proving the sufficiency for $\gamma=\infty$. Now suppose that $\gamma<\infty$. Since $w$ is non-increasing and $i_{1}<\ldots<i_{r}$, we have the following inequalities:

$$
\begin{aligned}
& \sum_{i_{k} \leq 2^{m} k} \beta^{k}\left(\int_{\left(i_{1}-1\right) \xi}^{i_{1} \xi} w+\ldots+\int_{\left(i_{k}-1\right) \xi}^{i_{k} \xi} w\right) \\
& \geq \sum_{\substack{i_{k} \leq 2^{m} k \\
\left(2^{m}+1\right) k \xi<\gamma}} \beta^{k}\left(\int_{2^{m} k \xi}^{\left(2^{m} k+1\right) \xi} w+\ldots+\int_{2^{m} k \xi}^{\left(2^{m} k+1\right) \xi} w\right) \\
& +\sum_{\substack{i_{k} \leq 2^{m} k \\
\left(2^{m}+1\right) k \xi \geq \gamma}} \beta^{k}\left(\int_{\left(i_{1}-1\right) \xi}^{i_{1} \xi} w+\ldots+\int_{\left(i_{k}-1\right) \xi}^{i_{k} \xi} w\right) \\
& \geq \sum_{\substack{i_{k} \leq 2^{m} k \\
\left(2^{m}+1\right) k \xi<\gamma}} k \beta^{k} \int_{2^{m} k \xi}^{\left(2^{m} k+1\right) \xi} w+\sum_{\substack{i_{k} \leq 2^{m} k \\
\left(2^{m}+1\right) k \xi \geq \gamma}} \beta^{k}\left(\int_{\gamma-k \xi}^{\gamma-(k-1) \xi} w+\ldots+\int_{\gamma-\xi}^{\gamma} w\right) \\
& \geq \sum_{\substack{i_{k} \leq 2^{m} k \\
\left(2^{m}+1\right) k \xi<\gamma}} k \beta^{k} \int_{2^{m} k \xi}^{\left(2^{m} k+1\right) \xi} w+\sum_{\substack{i_{k} \leq 2^{m} k \\
\left(2^{m}+1\right) k \xi \geq \gamma}} \beta^{k} \int_{\gamma-k \xi}^{\gamma} w .
\end{aligned}
$$

Then by (3.2) there is $\theta>0$ depending only on $m$ and such that 


$$
\sum_{i_{k} \leq 2^{m} k} \beta^{k}\left(\int_{\left(i_{1}-1\right) \xi}^{i_{1} \xi} w+\ldots+\int_{\left(i_{k}-1\right) \xi}^{i_{k} \xi} w\right)
$$

$$
\geq \sum_{\substack{i_{k} \leq 2^{m} k \\\left(2^{m}+1\right) k \xi<\gamma}} k \beta^{k} \int_{2^{m} k \xi}^{\left(2^{m} k+1\right) \xi} w+\sum_{\substack{i_{k} \leq 2^{m} k \\\left(2^{m}+1\right) k \xi \geq \gamma}} \beta^{k} \int_{\gamma-k \xi}^{\gamma} w
$$

$$
\geq \sum_{\substack{i_{k} \leq 2^{m} k \\\left(2^{m}+1\right) k \xi<\gamma}} \beta^{k} \int_{2^{m} k \xi}^{\left(2^{m}+1\right) k \xi} w+\sum_{\substack{i_{k} \leq 2^{m} k \\\left(2^{m}+1\right) k \xi \geq \gamma}} \beta^{k} \int_{\gamma-k \xi}^{\gamma} w
$$

$$
\geq \sum_{\substack{i_{k} \leq 2^{m} k \\\left(2^{m}+1\right) k \xi<\gamma}} \theta \beta^{k} \int_{0}^{k \xi} w+\sum_{\substack{i_{k} \leq 2^{m} k \\ \gamma /\left(2^{m}+1\right) \leq k \xi \leq \gamma}} \beta^{k} \int_{\gamma-k \xi}^{\gamma} w,
$$

and by (3.3), we find $\theta_{1}>0$ depending only on $m$ so that

$$
\begin{aligned}
\sum_{i_{k} \leq 2^{m} k} \beta^{k}\left(\int_{\left(i_{1}-1\right) \xi}^{i_{1} \xi} w+\ldots+\int_{\left(i_{k}-1\right) \xi}^{i_{k} \xi} w\right) & \sum_{\substack{i_{k} \leq 2^{m} k \\
\left(2^{m}+1\right) k \xi<\gamma}} \theta \beta^{k} \int_{0}^{k \xi} w+\sum_{\substack{i_{k} \leq 2^{m} k \\
\gamma /\left(2^{m}+1\right) \leq k \xi \leq \gamma}} \beta^{k} \int_{\gamma-k \xi}^{\gamma} w \\
\geq & \sum_{\substack{i_{k} \leq 2^{m} k \\
\left(2^{m}+1\right) k \xi<\gamma}} \theta \beta^{k} \int_{0}^{k \xi} w+\sum_{\substack{i_{k} \leq 2^{m} k \\
\gamma /\left(2^{m}+1\right) \leq k \xi \leq \gamma}} \theta_{1} \beta^{k} \int_{0}^{k \xi} w \\
\geq & \min \left\{\theta, \theta_{1}\right\} \sum_{i_{k} \leq 2^{m} k} \beta^{k} \int_{0}^{k \xi} w \frac{\min \left\{\theta, \theta_{1}\right\} \varepsilon_{3}}{2} .
\end{aligned}
$$

Again this contradicts (3.10) and the sufficiency is proved.

Conversely, if $\Lambda_{\varphi, w}$ is uniformly $c$-convex, then it is clearly complex rotund, and by Corollary 2.2, $\varphi$ satisfies the $\Delta_{2}^{w}$-condition. Notice also that a Banach space which contains $\ell_{\infty}^{n}$ 's $\lambda$-uniformly for all $\lambda>1$ cannot be uniformly $c$-convex. Hence, in view of Theorem 1.4, $w$ is regular. The proof is complete.

THEOREM 3.5. Let $\Lambda_{\varphi, w}$ be a complex Orlicz-Lorentz space. The following conditions are equivalent.

(1) $\Lambda_{\varphi, w}$ is uniformly c-convex.

(2) $\varphi$ satisfies the condition $\Delta_{2}^{w}$ and the weight function $w$ is regular. 
(3) $\Lambda_{\varphi, w}$ is of cotype $q$ for some $2 \leq q<\infty$.

(4) $\Lambda_{\varphi, w}$ has an equivalent $q$-uniformly $P L$-convex norm for some $2 \leq$ $q<\infty$.

(5) $\Lambda_{\varphi, w}$ is uniformly PL-convex.

Proof. By Theorem 3.4 we know that (1) is equivalent to (2). The equivalences $(3) \Leftrightarrow(4)$ and $(1) \Leftrightarrow(5)$ have been proved in Corollaries 7.1 and 7.4 of [7] and Theorem 2.1 of [9], respectively. If (1) is satisfied then $\Lambda_{\varphi, w}$ cannot contain $\ell_{\infty}^{n}$ 's $\lambda$-uniformly for all $\lambda \geq 1$, and by the well known result of Pisier (Theorem 14.1 in [8]), $\Lambda_{\varphi, w}$ has finite cotype, and so (3) is satisfied.

Finally, we will show that $(3) \Rightarrow(2)$. Suppose that $(2)$ is not true. If $\varphi$ does not satisfy the $\Delta_{2}^{w}$-condition, then by Theorem $1.3, \Lambda_{\varphi, w}$ contains an isometric copy of $\ell_{\infty}$, and so it cannot be of finite cotype. If $w$ is not regular, then by Theorem 1.4, $\Lambda_{\varphi, w}$ contains $\ell_{\infty}^{n}$ 's $\lambda$-uniformly for all $\lambda>1$, and again by Pisier's result, it cannot have any finite cotype.

The next result on uniform $c$-convexity in Lorentz spaces is an immediate corollary of the previous theorem.

Corollary 3.6. Let $\Lambda_{p, w}, 1 \leq p<\infty$, be a complex Lorentz space. The following conditions are equivalent.

(1) $\Lambda_{p, w}$ is uniformly c-convex.

(2) The weight function $w$ is regular.

(3) $\Lambda_{p, w}$ is of cotype $q$ for some $2 \leq q<\infty$.

(4) $\Lambda_{p, w}$ has an equivalent q-uniformly $P L$-convex norm for some $2 \leq$ $q<\infty$.

(5) $\Lambda_{p, w}$ is uniformly $P L$-convex.

4. Applications to norm-attaining operators. Let $\{w(n)\} \in c_{0} \backslash \ell_{1}$ be a decreasing sequence of positive numbers, and let $\Psi(n)=\sum_{k=1}^{n} w(k)$. For a complex sequence $x=\{x(n)\}$, define the functional

$$
\|x\|_{\Psi}=\sup _{n \geq 1} \frac{\sum_{k=1}^{n} x^{*}(k)}{\Psi(n)},
$$

where $\left\{x^{*}(n)\right\}$ is a decreasing rearrangement of $x=\{x(n)\}$. The Marcinkiewicz sequence space $m_{\Psi}$ is the space of all sequences $x$ with $\|x\|_{\Psi}<\infty$. Its order continuous subspace $d_{*}(w, 1)=m_{\Psi}^{0}$ is the subspace of $m_{\Psi}$ consisting of all $x=\{x(n)\}$ with

$$
\lim _{n \rightarrow \infty} \frac{\sum_{k=1}^{n} x^{*}(k)}{\Psi(n)}=0 .
$$

It is well known $[14,18,20]$ that $d_{*}(w, 1)$ is a predual of the Lorentz sequence space $d(w, 1)$ equipped with the norm \|\|$_{w, 1}$. 
Let $X$ and $Y$ be Banach spaces, and let $L(X, Y)$ be the space of all bounded linear operators from $X$ to $Y$, and $N A(X, Y) \subset L(X, Y)$ be the set of all norm-attaining operators. Recall that a bounded operator $T: X \rightarrow Y$ is norm-attaining if $\|T x\|=\|T\|$ for some $x \in B_{X}$. When $Y=\mathbb{R}$, Bishop and Phelps [4] showed that $N A(X, Y)$ is dense in $L(X, Y)$. Since then there have been many attempts to prove or disprove for what $X$ or $Y, N A(X, Y)$ is dense in $L(X, Y)$. In [11], Gowers showed that $N A\left(G, \ell_{p}\right), 1<p<\infty$, is not dense in $L\left(G, \ell_{p}\right)$, where $G=d_{*}(w, 1)$ and $w=\left\{n^{-1}\right\}_{n=1}^{\infty}$. Using the same space $G$, the authors of [1] showed that $N A\left(G, G^{*}\right)$ is not dense in $L\left(G, G^{*}\right)$ if $G$ is equipped with a proper equivalent norm. They also raised the question whether this is also true for $G$ with its original norm. Here we answer their question in the affirmative for complex spaces $G$. Let us start with the following elementary lemma.

Lemma 4.1. The identity operator $I: d_{*}(w, 1) \rightarrow d(w, 1)$ is a bounded operator if and only if the weight sequence $\{w(n)\}$ is in $\ell_{2}$.

Proof. Suppose that the identity operator $I: d_{*}(w, 1) \rightarrow d(w, 1)$ is bounded. Recall that $d(w, 1)$ is a Köthe dual of $d_{*}(w, 1)([20,18])$, that is,

$$
\|x\|_{w, 1}=\sup \left\{\langle x, y\rangle=\sum_{n=1}^{\infty} x(n) y(n):\|y\|_{\Psi} \leq 1\right\} .
$$

Hence for each $n \geq 1$, taking $x_{n}=\sum_{k=1}^{n} w(k) e_{k}$, where $e_{k}$ are the standard unit vectors, we have $\left\|x_{n}\right\|_{\Psi} \leq 1$ and so,

$$
\left\langle I x_{n}, x_{n}\right\rangle=\sum_{k=1}^{n} w(k)^{2} \leq\|I\| .
$$

Thus $\{w(n)\} \in \ell_{2}$.

For the converse, suppose that $\{w(n)\} \in \ell_{2}$. By definition of \|\|$_{\Psi}$, for any $x \in d_{*}(w, 1)$ and each $n \in \mathbb{N}$,

$$
\sum_{k=1}^{n} x^{*}(k) \leq\|x\|_{\Psi} \Psi(n) .
$$

Now, by summation by parts, if we set $\Psi(0)=0$ and $S(n)=\sum_{k=1}^{n} x^{*}(k)$ for each $n \in \mathbb{N}$ and $S(0)=0$, then

$$
\begin{aligned}
\sum_{k=1}^{n} x^{*}(k) w(k) & =\sum_{k=1}^{n}(S(k)-S(k-1)) w(k) \\
& =\sum_{k=1}^{n-1} S(k)(w(k)-w(k+1))+S(n) w(n)
\end{aligned}
$$




$$
\begin{aligned}
& \leq\|x\|_{\Psi} \sum_{k=1}^{n-1} \Psi(k)(w(k)-w(k+1))+\|x\|_{\Psi} \Psi(n) w(n) \\
& \leq\|x\|_{\Psi} \sum_{k=1}^{n}(\Psi(k)-\Psi(k-1)) w(k)=\|x\|_{\Psi} \sum_{k=1}^{n} w(k)^{2} \leq\|x\|_{\Psi} \sum_{k=1}^{\infty} w(k)^{2} .
\end{aligned}
$$

Therefore the identity operator $I$ is well defined and bounded. This completes the proof.

Recall the result $([13,18,14])$ characterizing the geometric structure of the ball in Marcinkiewicz spaces.

Lemma 4.2. For each $x \in B_{d_{*}(w, 1)}$, there exist $n \in \mathbb{N}$ and $\varepsilon>0$ such that $\|x+\lambda y\| \leq 1$ for each $y \in B_{d_{*}(w, 1)}$ with $y=(0, \ldots, 0, y(n+1), \ldots)$, and for each $|\lambda| \leq \varepsilon$.

Theorem 4.3. Let $d(w, 1)$ and $d_{*}(w, 1)$ be complex spaces. Then the set $N A\left(d_{*}(w, 1), d(w, 1)\right)$ is not dense in $L\left(d_{*}(w, 1), d(w, 1)\right)$ if $\{w(n)\} \in \ell_{2}$.

Proof. First we shall show that every norm-attaining operator from $d_{*}(w, 1)$ into $d(w, 1)$ has a finite rank. Suppose $T \in N A\left(d_{*}(w, 1), d(w, 1)\right)$ and $\|T\|=\left\|T x_{0}\right\|_{w, 1}$ for $x_{0} \in B_{d_{*}(w, 1)}$. By Lemma 4.2, there are $n \geq 1$ and $\varepsilon>0$ such that for each $y=(0, \ldots, 0, y(n+1), \ldots) \in B_{d_{*}(w, 1)}$ and for each $|\zeta|<\varepsilon$,

$$
\left\|x_{0}+\zeta y\right\|_{\Psi} \leq 1
$$

Hence

$$
\|T\|=\left\|T x_{0}\right\|_{w, 1} \geq\left\|T\left(x_{0}+\zeta y\right)\right\|_{w, 1}=\left\|T x_{0}+\zeta T y\right\|_{w, 1} .
$$

Notice that the complex Lorentz space $d(w, 1)$ is complex rotund by Corollary 2.6. Therefore the strong maximum modulus theorem holds in $d(w, 1)$ (see [22]). This implies that $\left\|T x_{0}+\zeta T y\right\|_{w, 1}$ is constant for every $|\zeta|<\varepsilon$, and so $T y=0$. Thus every norm-attaining operator has a finite rank. Now, for any $T \in N A\left(d_{*}(w, 1), d(w, 1)\right)$ there exists $n \in \mathbb{N}$ such that $T e_{m}=0$ for all $m>n$. Hence

$$
\|I-T\| \geq w(1)\left\|e_{m}\right\|_{w, 1}=w(1)^{2}>0 .
$$

Thus $N A\left(d_{*}(w, 1), d(w, 1)\right)$ is not dense in $L\left(d_{*}(w, 1), d(w, 1)\right)$, and this completes the proof.

In fact we can say more. In [14], it is proved that the condition $w \in \ell_{2}$ holds if and only if the space $A L^{2}\left(d_{*}(w, 1)\right)$ of norm-attaining continuous bilinear forms is not dense in the space $L^{2}\left(d_{*}(w, 1)\right)$ of continuous bilinear forms on $d_{*}(w, 1)$. If we identify, in a natural way, the space $L^{2}\left(d_{*}(w, 1)\right)$ with $L\left(d_{*}(w, 1), d(w, 1)\right)$, then $A L^{2}\left(d_{*}(w, 1)\right) \subset N A\left(d_{*}(w, 1), d(w, 1)\right)$ (see [1]). 
This implies that if $w \in c_{0} \backslash \ell_{2}$, then $N A\left(d_{*}(w, 1), d(w, 1)\right)$ is dense in $L\left(d_{*}(w, 1), d(w, 1)\right)$. Summarizing these facts we obtain the following result.

Corollary 4.4. Let $d(w, 1)$ and $d_{*}(w, 1)$ be complex spaces. Then the set $N A\left(d_{*}(w, 1), d(w, 1)\right)$ is dense in $L\left(d_{*}(w, 1), d(w, 1)\right)$ if and only if $\{w(n)\} \notin \ell_{2}$.

\section{References}

[1] M. D. Acosta, F. J. Aguirre and R. Payá, There is no bilinear Bishop-Phelps theorem, Israel J. Math. 93 (1996), 221-227.

[2] Z. Altshuler, Uniform convexity in Lorentz sequence spaces, ibid. 20 (1975), 260-274.

[3] C. Bennett and R. Sharpley, Interpolation of Operators, Academic Press, 1988.

[4] E. Bishop and R.R. Phelps, A proof that every Banach space is subreflexive, Bull. Amer. Math. Soc. 67 (1961), 97-98.

[5] J. Cerdá, H. Hudzik, A. Kamińska and M. Mastyło, Geometric properties of symmetric spaces with applications to Orlicz-Lorentz spaces, Positivity 2 (1998), 311-337.

[6] S. T. Chen, Geometry of Orlicz Spaces, Dissertationes Math. 356 (1996).

[7] W. Davis, D. J. H. Garling and N. Tomczak-Jaegermann, The complex convexity of quasi-normed linear spaces, J. Funct. Anal. 55 (1984), 110-150.

[8] J. Diestel, H. Jarchow and A. Tonge, Absolutely Summing Operators, Cambridge Univ. Press, 1995.

[9] S. J. Dilworth, Complex convexity and the geometry of Banach spaces, Math. Proc. Cambridge Philos. Soc. 99 (1986), 495-506.

[10] J. Globevnik, On complex strict and uniform convexity, Proc. Amer. Math. Soc. 47 (1975), 175-178.

[11] W. T. Gowers, Symmetric block bases of sequences with large average growth, Israel J. Math. 69 (1990), 129-151.

[12] I. Halperin, Uniform convexity in function spaces, Duke Math. J. 21 (1954), 195-204.

[13] K. H. Han and H. G. Song, Extensions of polynomials on preduals of Lorentz sequence spaces, preprint, 2002.

[14] M. Jiménez Sevilla and R. Payá, Norm-attaining multilinear forms and polynomials on preduals of Lorentz sequence spaces, Studia Math. 127 (1998), 99-112.

[15] A. Kamińska, Extreme points in Orlicz-Lorentz spaces, Arch. Math. (Basel) 55 (1990), 173-180.

[16] -, Some remarks on Orlicz-Lorentz spaces, Math. Nachr. 147 (1990), 29-38.

[17] - Uniform convexity of generalized Lorentz spaces, Arch. Math. (Basel) 56 (1991), 181-188.

[18] A. Kamińska and H. J. Lee, On uniqueness of extension of homogeneous polynomials, preprint, 2003.

[19] A. Kamińska, P. K. Lin and H. Sun, Uniform normal structure of Orlicz-Lorentz spaces, in: Interaction Between Functional Analysis, Harmonic Analysis and Probability, Dekker, 1996, 229-238.

[20] S. G. Kreĭn, Yu. I. Petunin and E. M. Semenov, Interpolation of Linear Operators, Transl. Math. Monogr. 54, Amer. Math. Soc., 1982.

[21] J. Musielak, Orlicz Spaces and Modular Spaces, Lecture Notes in Math. 1034, Springer, 1983. 
[22] E. Thorp and R. Whitley, The strong maximum modulus theorem for analytic functions into a Banach space, Proc. Amer. Math. Soc. 18 (1967), 640-646.

Changsun Choi and Han Ju Lee

Division of Applied Mathematics

Korea Advanced Institute

of Science and Technology

373-1, Kusong-Dong, Yusong-Gu

Taejon, 305-701, Republic of Korea

E-mail: choics@amath.kaist.ac.kr

hjlee@amath.kaist.ac.kr
Anna Kamińska

Department of Mathematical Sciences

The University of Memphis

Memphis, TN 38152, U.S.A.

E-mail: kaminska@memphis.edu

Received June 23, 2003 